\title{
Phalloplasty: techniques and outcomes
}

\author{
Aaron L. Heston ${ }^{1}$, Nick O. Esmonde ${ }^{1}$, Daniel D. Dugi III ${ }^{2}$, Jens Urs Berli ${ }^{1}$ \\ ${ }^{1}$ Division of Plastic and Reconstructive Surgery, ${ }^{2}$ Division of Urology, Oregon Health \& Science University, Portland, Oregon, USA \\ Contributions: (I) Conception and design: All authors; (II) Administrative support: None; (III) Provision of study material or patients: None; (IV) \\ Collection and assembly of data: AL Heston, NO Esmonde, JU Berli; (V) Data analysis and interpretation: AL Heston, NO Esmonde, JU Berli; (VI) \\ Manuscript writing: All authors; (VII) Final approval of manuscript: All authors. \\ Correspondence to: Jens Urs Berli, MD. Assistant Professor, Division of Plastic \& Reconstructive Surgery, Transgender Health Program, Oregon \\ Health \& Science University, 3181 SW Sam Jackson Park Road, MAC 3168C, Portland, Oregon 97239, USA. Email: berli@ohsu.edu.
}

\begin{abstract}
Phalloplasty is an exceptionally complicated reconstructive procedure that attempts to create a structure that is penis-like. As patient goals vary widely, it is helpful to think about phalloplasty as a modular set of procedures that can be combined, mixed and matched to meet the needs of each individual patient while also taking into account their anatomy. Each module-but particularly the shaft and penile urethracan be performed using a variety of techniques. To date, there is no consensus among surgeons regarding the optimum staging of the reconstructive steps. Our primary goal is to outline the most frequently performed and reported options in phallic reconstruction and outline the various considerations that go into choosing a given sequence of procedures for the specific patient. The secondary goal of this article is to describe the complications common to each of those modules and how they interact when combined.
\end{abstract}

Keywords: Phalloplasty; gender confirmation surgery (GCS); reconstructive surgical methods; review; surgical flaps; penis/surgery

Submitted Nov 28, 2018. Accepted for publication May 06, 2019.

doi: $10.21037 /$ tau.2019.05.05

View this article at: http://dx.doi.org/10.21037/tau.2019.05.05

\section{Introduction}

The word phallus comes from Greek and means penislike. In art, it is often used to refer to symbols that resemble the erect male genitalia. Hence, phalloplasty is a plastic surgery operation that attempts to create a structure that is penis-like. The article will focus on surgery as part of the treatment for gender dysphoria in trans male patients.

The goals of phalloplasty have been described previously as achieving some or all of the following goals (1):

* Aesthetic appearing phallus;

* Tactile and erogenous sensation;

* Standing micturition;

* Ability to have an erection and penetrative intercourse.

As the female and male genitals share a common embryologic origin, gender confirmation surgery (GCS) involving the genitals strives to utilize analogous structures to produce a proper cosmetic result. This principle is used in vaginoplasty for trans female patients and commonly results in a both an aesthetically and functionally satisfying result.

Metoidioplasty attempts to use similar principles by releasing the tethering of the clitoris to the pubic bone and urethra, respectively, as well as optionally extending the urethra to the glans (2). Unfortunately, this reconstructive option falls short in several of the above domains. Standing micturition is not always possible, and especially when attempted through the pant zipper. The clitoral tissue retains its erectile qualities, but the result is frequently too short to allow for penetrative intercourse. Finally, the aesthetic outcome is unsatisfactory when compared to a cismale penis and does not allow for patients to comfortably "pass" in a locker room environment. Phalloplasty is thus the preferred option for many trans male patients.

Phalloplasty is an exceptionally complicated reconstructive procedure with various techniques described, but there is 
no agreed upon gold standard. Rather, there are several commonly used flaps available and different groups have advocated varying ways to stage and sequence them. To date, there is no consensus among GCS surgeons regarding the optimum staging or sequencing of the reconstructive steps.

\section{Modular concept}

Phalloplasty is not a homogenous procedure with simple variations. Rather, it is a patient and surgeon-specific combination of the following sub procedures that are used to meet the patient's goals:

* Phallic shaft creation;

* Penile urethroplasty;

* Perineal urethroplasty (a.k.a. urethral lengthening);

* Perineoplasty;

* Scrotoplasty;

* Vaginectomy;

* Hysterectomy \& oophorectomy;

* Glansplasty;

* Testicular implants;

* Erectile device.

It is helpful to use the above list to think about phalloplasty as a modular set of procedures that can be combined, mixed and matched to meet the needs of each individual patient. Each module-but particularly the shaft and penile urethra — can be performed using a variety of techniques. Because of the individualized nature of phalloplasty, a comprehensive review is not possible in just one article. Our primary goal is to outline the most frequently performed and reported options in phallic reconstruction and outline to the reader the various considerations that go into choosing a given sequence of procedures for the specific patient. This also must include consideration of the patient's social circumstances, support network, medical comorbidities, institutional resources, insurance status, and financial resources.

The modular concept also illustrates why the complication rate in this surgery is high. Each module comes with its own potential complications and the complications are additive. Therefore we stage the surgery as to mitigate the effects of individual complications on the overall phallic reconstruction. For example, a simple wound separation can be devastating if the urinary system has already been completed, whereas if the surgery is staged it can be managed more simply. The secondary goal of this article is that surgeons performing phalloplasty become familiar with the complications common to each of those modules and how they interact when combined.

A successful phalloplasty program involves the ability to manage the frequent occurrence of complications. This is best achieved by having a multidisciplinary team involving a reconstructive urologist and plastic surgeons. In the experience of the authors, the patients are largely aware of the risks involved and when proper informed consent has taken place are very informed and understanding of the potential for complications.

\section{Fundamental urologic choices before planning surgery}

Before choosing a donor site or reconstructive plan the patient should decide if urinating from the tip of the phallus is imperative. We believe this is an essential branch point in the decision tree. Some patients may accept sitting urination and this decision means that a shaft-only phallus can be created and thus reduces the risk profile.

If a patient opts to forgo urination from the tip of the phallus, then there are a few other important decision points. The urethra can either be left in its native position or lengthened to the base of the phallus using labia minora tissue. This type of reconstruction would allow later secondary urethroplasty should the patient desire this as part of their ongoing transition. We recommend however that it be left in its native position since that has the lowest risk profile and also avoids any potential issues of spraying and unpredictable urinary stream direction. If left in the native position, the patient can still decide if they desire scrotoplasty +/- vaginectomy. If a vaginectomy and perineoplasty is performed, then the urethra is externalized as a perineal urostomy.

For patients that desire phallic urination, the native urethra needs to be lengthened to create a perineal urethra that can be connected to the penile urethra within the shaft. Most surgeons recommend a vaginectomy whenever the urethra is lengthened to cover the urethroplasty with various vascularized layers. In a review of urethral lengthening techniques, Massie et al. noted that vaginectomy is associated with a significant decrease in complications because it allows additional vascularized bulbospongiosus muscle to cover the urethroplasty suture line (3). Without vaginectomy, only labia minora tissue is available for urethral lengthening but can be augmented with vaginal mucosa tissue proximal to the urethroplasty suture line and fascio-lipomatous tissue at the distal suture 
Table 1 Shaft creation options and the corresponding uro/gyn requirements and possible shaft donor sites

\begin{tabular}{llll}
\hline & Shaft only & Tube within a tube & Composite shaft \\
\hline Shaft donor sites & ALT, RFFF, abdominal, SCIP, TdAP & RFFFP, ALT, fibula & RFFFP, ALT, latissimus dorsi \\
Penile urethra & None & Sourced with shaft flap & Radial forearm, ulnar forearm, groin \\
Native urethral lengthening & $\begin{array}{l}\text { (I) None; (II) to base of shaft; (III) } \\
\text { perineal urostomy }\end{array}$ & Required & Required \\
Vaginectomy & Optional & Required & Required \\
Hysterectomy & Optional & Required & Required \\
\hline
\end{tabular}

ALT, anterolateral-thigh; RFFF, radial forearm free flap; SCIP, superficial circumflex iliac artery perforator flap; TdAP, thoracodorsal artery preforator flap.

line. Additionally, a pedicled gracilis muscle can be used to provide yet another well vascularized layer of tissue (4).

\section{Preoperative exam}

The ideal donor site should be thin and relatively hairless. If the donor site contains hair, then hair removal via laser or electrolysis should be initiated. Any exam of scars from either self-harm or previous injuries should be noted and may preclude a donor site as they may compromise a reliable blood supply.

Beyond the health of the donor site, patient selection is important to avoid complications. There is no objective data to guide the risk profile of any given patient, but it is the senior authors opinion that a BMI less than 30 is ideal and a BMI greater than 35 is a relative contraindication due to increased distance to recipient structures and thickness of donor sites. However, skin laxity and body fat distribution may be more important to consider than an absolute $\mathrm{BMI}$ value. Truncal obesity is a potential contraindication because of added postoperative risk. All patients should be counseled for smoking cessation and weight loss prior to surgery. We find that most patients are eager to make lifestyle choices to improve their chances of an optimal outcome.

The importance of a psychosocial assessment pre-op cannot be overstated. The patient must have a demonstrable postoperative care plan in place. Phalloplasty surgery has enormous consequences on the patient's mobility, ability to work, ability to socialize, ability to travel, housing and bathing, intimacy with their partners, in addition to frequent post-operative appointments during the first 1-2 months after surgery. These points must be emphasized pre-operatively. Compliance and health literacy are important factors to assess. At our institution, we require patients and their caregiver attend a pre-operative education class that is managed by the surgeons and the transgender healthcare social worker. This educational process is a continuous team effort while in the hospital and after discharge.

\section{Choice of flap design and donor site}

Donor site choice and design are interdependent. Table 1 and Figure 1 outline various phallus designs and flap options. If the patient has decided to forgo urination from the tip of the phallus, then a shaft-only design will be used. A shaft only design is essentially a rectangular pattern flap with near equidistant length to width ratio. Figure 2 shows a variety of shaft only examples.

For phallic urination, several options exist. The most frequently used design is the so-called tube within a tube (TWT). The TWT design consists of two skin paddles that are rolled in opposite directions with an intervening dermal bridge for blood supply of the urethral skin paddle.

It is also possible to create a composite flap where the urethra and shaft are taken from separate donor sites. An example of this concept is a pedicled pedicled anterolateralthigh (ALT) shaft with a radial or ulnar forearm free flap urethra (5). This is a good option for patients who desire to minimize forearm scarring but have unsuitable thigh skin to allow for a TWT ALT. Alternatively, it may be useful if a patient has a very thin and small donor forearm that is inadequate for radial forearm phalloplasty. An example is shown in Figure 3.

Other surgeons prefer to construct the urethra by means of grafting-either in a staged prelamination method (i.e., vaginal/uterine mucosa around a catheter on forearm)or to split a healed shaft-only phalloplasty and line it with buccal graft, vaginal mucosa or skin graft. This is followed 
A

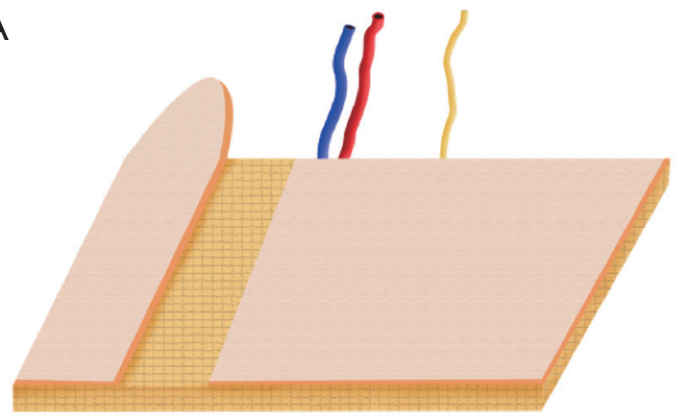

Tube within a tube

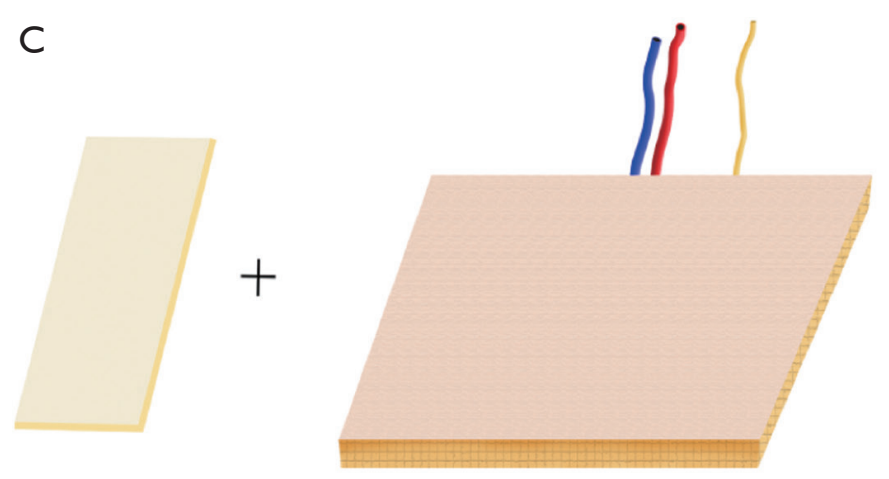

Vascularized shaft, grafted urethra

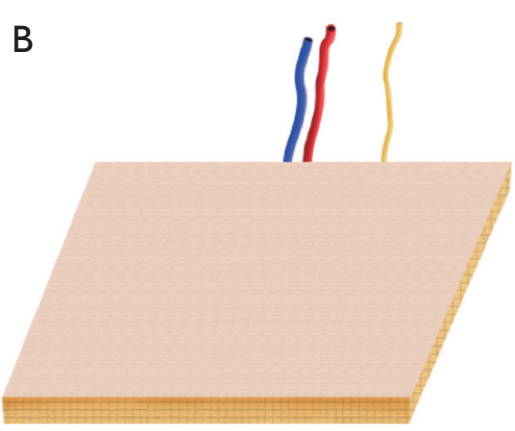

Shaft only

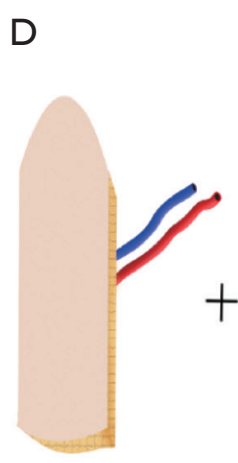

Composite

Figure 1 Illustration of various flap designs used for phalloplasty. Illustration copyright Jourdan Carboy/Jens Berli.

by tubularization at the next surgery (6).

The next question to decide with the patient is which donor site(s) to use. Table 2 summarizes for which purposes the commonly used phalloplasty flaps are suitable for. Historically, surgeons first started by using loco-regional pedicled flap options that were left attached to their source neuro-vascular bundle. With the advent of microsurgical techniques in the last century various distant sites including forearm, latissimus dorsi and fibula were utilized as free flaps. The last addition to the reconstructive options was the anterolateral-thigh (ALT) flap. In current practice, the most frequently used donor sites are the forearm and the ALT (7). The superficial circumflex iliac artery perforator flap (SCIP) is also gaining popularity used both for shaft and urethral reconstruction. While the physical exam guides which donor sites are viable options, the patient's preferences are ultimately the deciding factor. For example, the radial forearm leaves a large, conspicuous scar that some patients find cosmetically unappealing or socially stigmatizing.

From a surgeon's perspective, the radial forearm free flap (RFFF) lends itself particularly well due to the ample and expendable innervation. It is also thin and pliable and has reliable vascularity (see Figure 4). Because of this, radial foream free flap phalloplasties (RFFFP) are the most commonly performed type (7). Patients report high satisfaction with the cosmetic appearance and most are eventually able to urinate from the phallus (1). The donor site can be covered with a split thickness skin graft or alternatively by acellular dermal matrices followed by skin graft a few weeks later.

To evaluate for RFFFP viability, the Allen test should be performed to make sure the radial artery can be safely harvested. The forearm skin and subcutaneous tissue should not be too thin or else the phallus will appear very deflated. Similarly, a forearm circumference of $15 \mathrm{~cm}$ and above at the wrist is desired in the authors opinion.

The ALT is the second most commonly used in current practice. A surgical advantage of the ALT is that it can often be performed as a pedicled flap (8). Similar to the forearm, the ALT is well vascularized and sensate (lateral femoral 

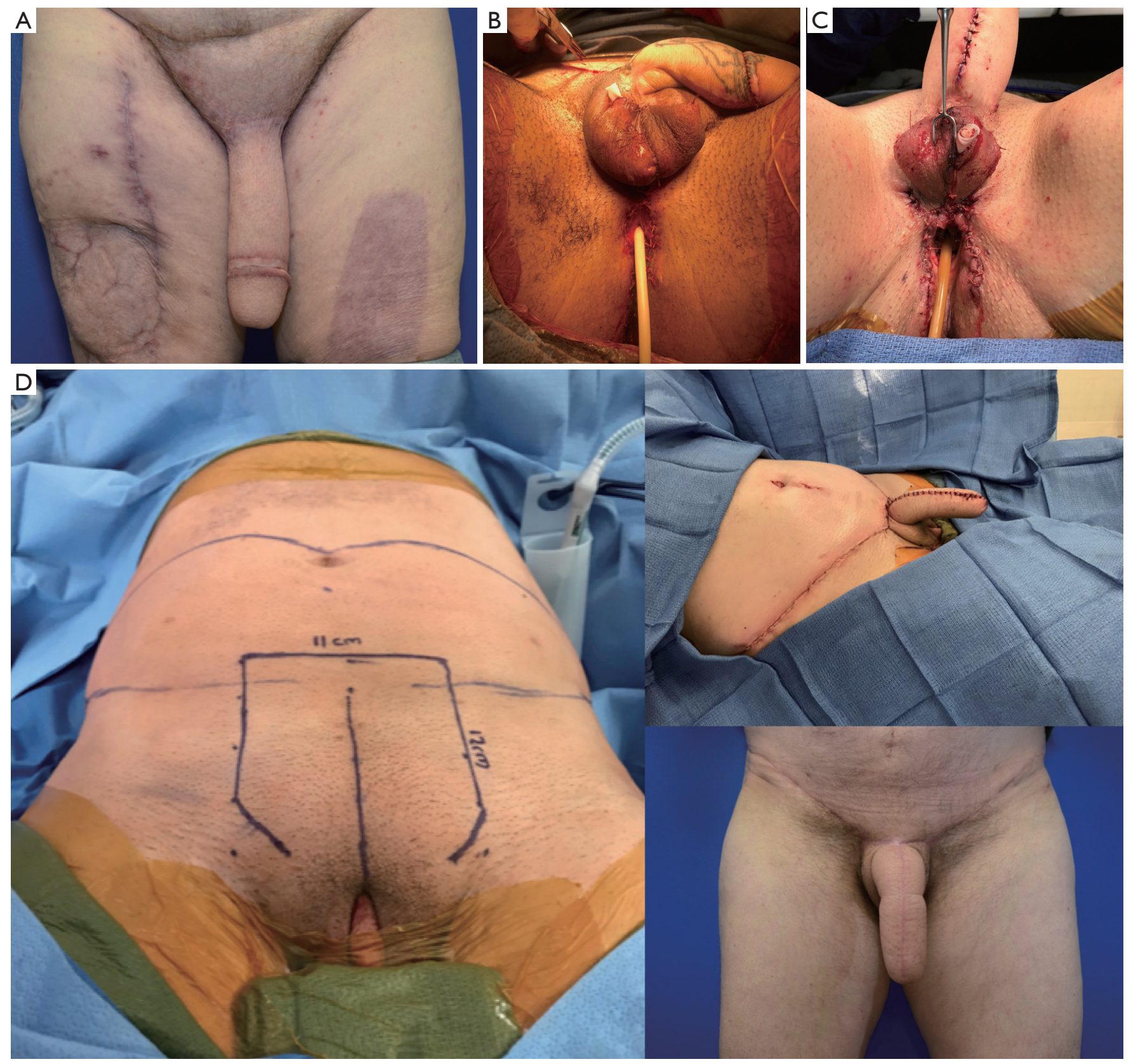

Figure 2 Examples of shaft only phalloplasty. (A) Shaft only phalloplasty without perineal surgery in an elderly trans male; (B) shaft only phalloplasty using a radial forearm flap and creation of perineal urostomy as well as scrotoplasty; (C) shaft only phalloplasty using a pedicled anterolateral thigh flap and creation of scrotum with retention of vaginal canal. Clitoral frenulum has been transected and moved inferiorly to create a superior vulvar commissure; (D) shaft only phalloplasty in a patient with previous metoidioplasty using an abdominal flap.

cutaneous nerve), and the safety of ALT flaps has been well documented in the literature (9). Patients may prefer ALT because it is easier to hide the donor scar and ability to create a longer phallus with more girth. Additionally, an ALT may allow for enough rigidity to allow for intercourse without the need for erectile device.

The flap, however, is frequently too bulky and the ultimate reconstruction-especially if performed as a TWT - is rather large. To evaluate for ALT viability, the thigh is examined for subcutaneous thickness at level of the 

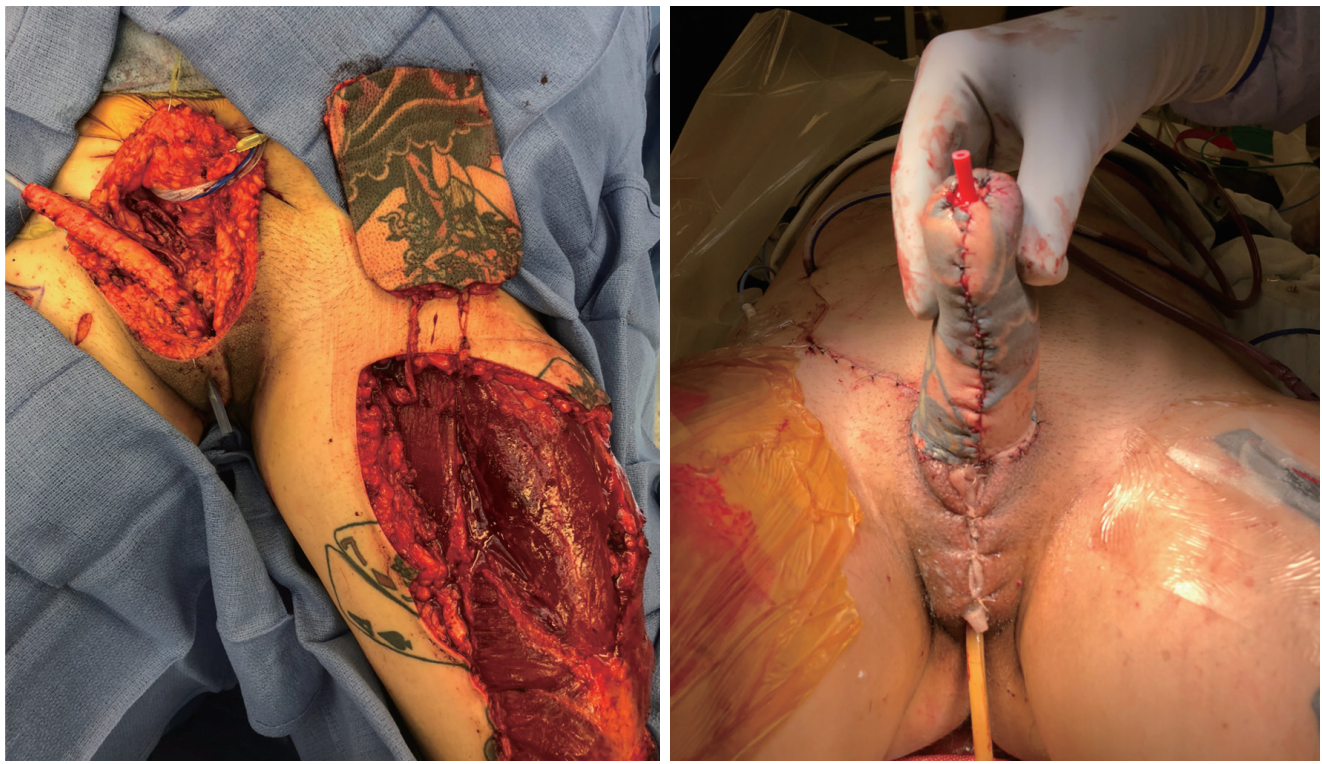

Figure 3 Composite phalloplasty using a free ulnar forearm flap for urethral creation and a pedicled anterolateral thigh flap for shaft creation.

Table 2 Commonly used flaps and their suitable applications in phalloplasty

\begin{tabular}{lccc}
\hline $\begin{array}{l}\text { Commonly used } \\
\text { donor sites }\end{array}$ & $\begin{array}{c}\text { Tube within } \\
\text { a tube }\end{array}$ & $\begin{array}{c}\text { Urethra } \\
\text { only }\end{array}$ & $\begin{array}{c}\text { Shaft } \\
\text { only }\end{array}$ \\
\hline Radial forearm & $\mathrm{X}$ & $\mathrm{X}$ & $\mathrm{X}$ \\
Ulnar forearm & $\mathrm{X}$ & $\mathrm{X}$ & $\mathrm{X}$ \\
ALT & & $\mathrm{X}$ \\
MLD & & $\mathrm{X}$ & $\mathrm{X}$ \\
Abdominal flap & & & $\mathrm{X}$ \\
SCIP & &
\end{tabular}

ALT, anterolateral-thigh; MLD, musculocutaneous latissimus dorsi; SCIP, superficial circumflex iliac artery perforator flap.

distal two thirds of the thigh. For a TWT design the thigh has to be extremely thin with a subcutaneous thickness of less than $1 \mathrm{~cm}$. For a shaft-only procedure (no urethral reconstruction) the thickness should not exceed $2-3 \mathrm{~cm}$, in our opinion. Due to the location of the nerves on top of the deep fascia, any debulking at the time of phalloplasty may result in damage to the phallic sensation.

Modified latissimus dorsi flaps are less common, but still in use. The donor site is well hidden and by means of tissue rearrangement can often be closed primarily. The flap is large but does not allow for a tube-within-a-tube penile urethra. This necessitates urethra lengthening by means of locoregional labial tissue and subsequent two-stage grafting of the urethra by means of buccal mucosa graft (10).

The free fibula flap seems appealing as it has internal rigidity and in theory abolishes the need for an erectile device. However, anchoring of the fibula to the pubic bone is problematic. Erosion is another issue that has made this a less desired flap choice.

\section{Choice of staging}

The next step in decision making is the staging. For the purposes of readability, we will refer to vaginectomy, urethral lengthening, perineoplasty and scrotoplasty as perineal masculinization.

Phalloplasties were historically performed as single stage procedures, with flap creation and perineal masculinization performed in one single major surgery. As discussed previously, this method is falling out of favor due to the additive risk of complications from each step involved. Most phalloplasties today are performed as staged procedures, with the tissue transfer and perineal masculinization performed months apart.

If, as the authors strongly suggest, you choose a vascularized option as penile urethral reconstruction then 

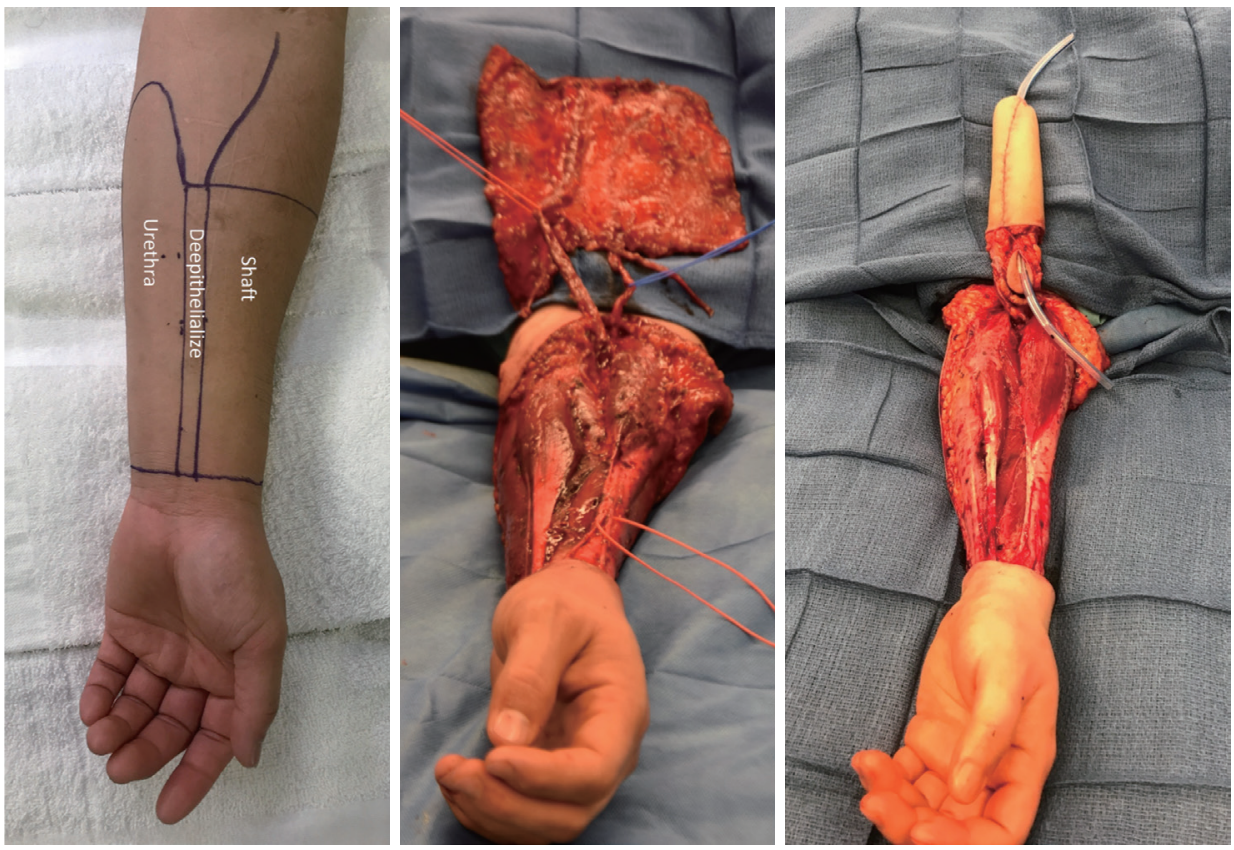

Figure 4 Radial forearm free flap phalloplasty using a tube within a tube flap design.

Table 3 Commonly used staging choices in phalloplasty

\begin{tabular}{|c|c|c|c|}
\hline Name & Staging choice & Advantage & Disadvantage \\
\hline "Single Stage" & $\begin{array}{l}\text { Stage 1: perineal masculinization } \\
\text { and creation of shaft and penile } \\
\text { urethra }\end{array}$ & Fewer surgeries & $\begin{array}{l}\text { Extensive anesthesia time. } \\
\text { Additive complications from } \\
\text { multiple complex procedures }\end{array}$ \\
\hline "Metoidio-First" & $\begin{array}{l}\text { Stage 1: perineoplasty (akin a } \\
\text { metoidioplasty). Stage } 2 \text { : creation } \\
\text { of shaft and urethra at the second } \\
\text { stage }\end{array}$ & $\begin{array}{l}\text { Patient may be ok with metoidioplasty and } \\
\text { not require further surgeries. Proximal urethral } \\
\text { anastomosis healed prior to second surgery }\end{array}$ & $\begin{array}{l}\text { Additional stage. Flap related } \\
\text { complications can have a } \\
\text { significant impact on the } \\
\text { patients' ability to urinate }\end{array}$ \\
\hline $\begin{array}{l}\text { "Big Ben Method" } \\
\text { (Developed by Dr. } \\
\text { Christopher Nim and } \\
\text { Dr. David Ralph in } \\
\text { London) }\end{array}$ & $\begin{array}{l}\text { Stage 1: creation of shaft and } \\
\text { penile urethra. Stage 2: perineal } \\
\text { masculinization }\end{array}$ & $\begin{array}{l}\text { Flap (i.e., small suture abscesses) can heal prior } \\
\text { to voidance. With theoretical benefit of lower } \\
\text { fistula rate. Suture line to connect the urethra } \\
\text { is not circumferential but longitudinal which } \\
\text { can be assumed leads to (fewer) strictures. In } \\
\text { case of flap failure the patient may continue to } \\
\text { void through native urethra and heal prior to } \\
\text { embarking on further reconstructions }\end{array}$ & $\begin{array}{l}\text { Additional stage. Ongoing } \\
\text { dysphoria from female } \\
\text { genitalia between stages }\end{array}$ \\
\hline
\end{tabular}

there are three choices for staging, as Table 3 outlines. There are pros and cons to each of them and also no truly comparative data to date that can definitively answer which is the best.

The same staging options apply for shaft-only patients, as some degree of perineal masculinization will be required unless the patient only wants a shaft with no scrotoplasty. An example of second stage perineal masculinization is shown in Figure 5.

Regardless of staging choice, erectile and testicular implants should only be inserted once the soft tissue reconstruction has healed.

The next most important step in successfully performing a phalloplasty is to have a streamlined perioperative process and adequately educate the patient and the care-team in the needed postoperative requirements. 

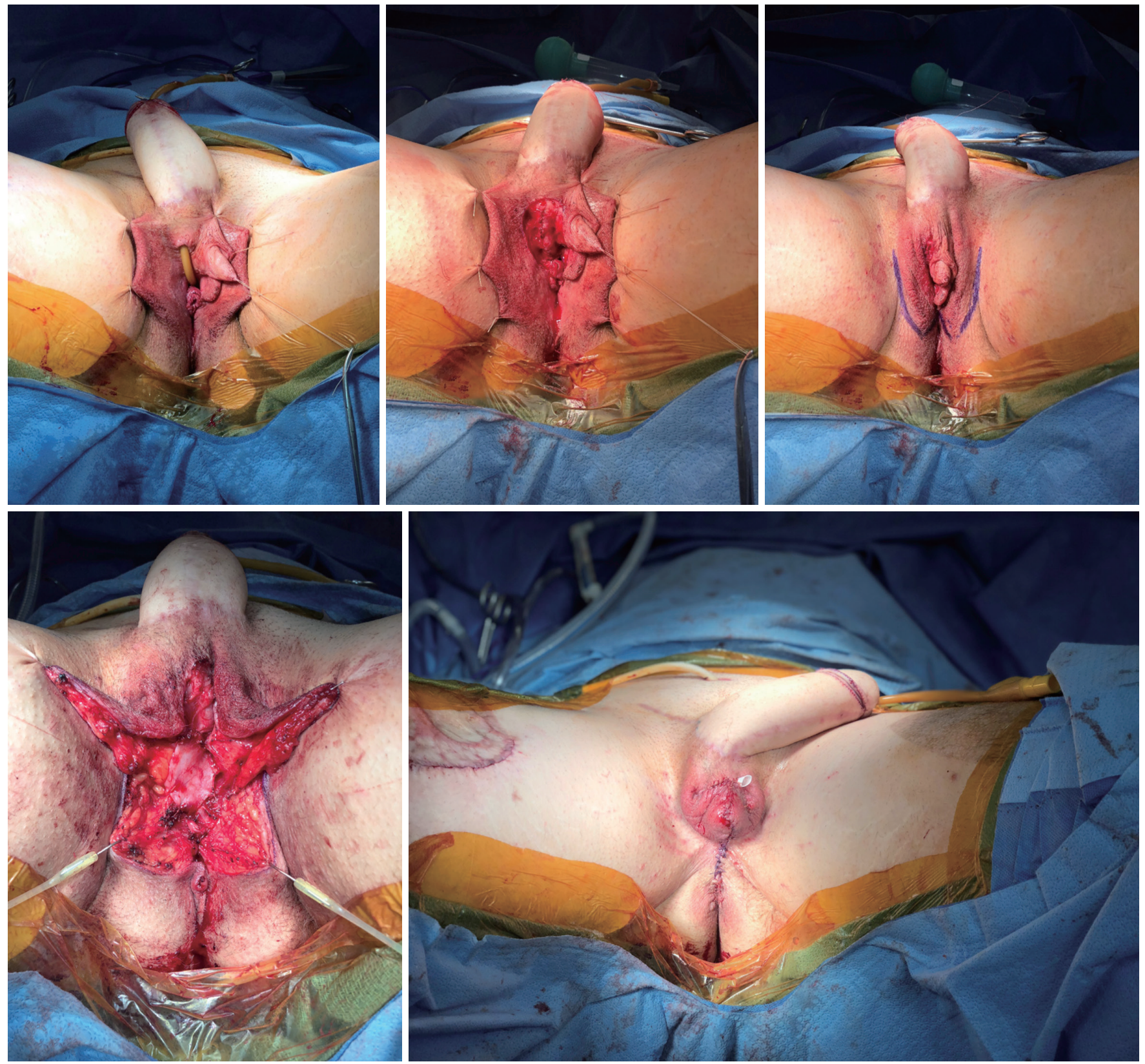

Figure 5 Second stage perineal masculinization with a pedicled ALT flap phallus. ALT, anterolateral-thigh.

\section{Management of flap related complications}

Even with excellent preparation and execution of the surgical plan, the gender surgeon is wise to anticipate one or more complications during phalloplasty reconstruction. We have published previously on this topic (11). While complications with phalloplasty are not a fait accompli, we encourage this mindset as it allows the surgeon to think more proactively and preventatively during the multiple reconstructive steps. Patient education around complications should similarly emphasize the near certainty of some degree of setback. Again, we advocate a staged approach to phalloplasty as the management of a small complication can quickly become overwhelming (for surgeon and for the patient/family) in the setting of managing the sequence of 
requisite procedures.

\section{Full phallic loss}

Phalloplasty is unique among microsurgical flaps in that the flap is freely attached to the integument and is tubularized around the pedicle in its final form. These conditions make this flap potentially susceptible to several different vascular insults which could compromise the flap. If the patient stands, the flap is then in a dependent position and easily susceptible to congestion. Similarly, flexion at the hip can obstruct inflow or outflow for the flap, depending on which vascular pedicle is used. It is therefore our recommendation to have the patient avoid bending at the waist beyond 20 degrees for four weeks post operatively. We allow for "essential ambulation" only during this time period. Also, we advocate for a dressing that allows the phallus to be at a 90-degree angle to the long axis of the patient's body, both during bedrest and while standing. This is important to prevent kinking of the flap or vascular pedicle.

We expect some degree of post-operative swelling within the flap, due to surgical trauma of flap elevation and transfer. Likewise, venous drainage may be slow to recover following free tissue transfer. We have seen several cases where swelling of the flap itself became compressive on the vascular pedicle and required bedside release of some of the ventral sutures to alleviate the pressure. We believe this is more common in the bulky ALT flap, but it can happen with RFFF-especially if there is a composite flap used for the urethra, which may be bulkier than the tube-in-tube design. This phenomenon can occur even with normal venous drainage. This phenomenon can be prevented by widening the base of the flap at the expense of a vertical straight raphe. We further require flap massage with each flap check, during which the nurse or doctor circumferentially compresses the flap from the meatus towards the base several times. We have anecdotally observed that this has reduced our incidence of critical flap swelling. For those flaps which required suture removal, we have managed those successfully with delayed primary closure.

Full flap loss is a rare but devastating event. We have offered several suggestions for how to reduce the risk, which is estimated to be $1.69 \%$ (7). For single stage reconstructions, the issues are compounded by the fact that the urethral lengthening makes it more difficult to convert the patient back to a conventional perineal urethra. To avoid this scenario, we promote a staged procedure, with urethral connection and perineal reconstruction performed at the second stage. With a staged approach, we can mitigate the risk of larger complications by breaking it down into relatively smaller sequence of procedures.

\section{Partial phallic loss}

Partial phalloplasty loss is one of the more common complications of phalloplasty. Monstrey et al. reported a $7.3 \%$ partial necrosis rate for the RFFFP in their large series of tube-in-tube constructs (12). The flap dimensions used for phalloplasty require a reliable pedicle and a healthy subdermal plexus to perfuse these broad flaps.

If the RFFFP TWT design is used, it is important to note that the radial portion of the flap is the most distal from the blood supply and presents a possible watershed area. This is an important consideration when positioning the flap design. Notably in an arm with a small circumference the radial vascular bundle may be too ulnar on the flap. The other area that is vulnerable is the neo-urethral extension and may suffer from insufficient blood supply. In our experience this area is more prone to ischemia when an ALT is used. For staged procedures, where the phallus is created prior to urethral lengthening, this is important to monitor, as any wound issues there would delay and/or complicate urethral lengthening at subsequent stages. For single staged procedures, there is no way to reliably monitor this vulnerable area in the acute post-operative period. Figure 6 shows a partial flap loss on a tattooed phallus.

For ALT reconstructions, we use CT angiography preoperatively to decide on the laterality of the flap. This allows us to identify the most distal perforator with the most favorable dissection course. We also use the scan to predict the pedicle length of the descending branch of the lateral femoral circumflex. In some cases, this may allow us to more accurately predict if a pedicled ALT flap is possible.

Management of partial flap loss follows basic reconstructive principles: debridement of devitalized tissues, control of infection, and reconstruction with grafts, dermal substitutes, or healing by secondary intention.

\section{Infection}

Infection is an inherently large concern due to the proximity of the flap to the groin, genitals, and rectum. Superficial infections along the external suture line are easily identified and respond well to treatment with first generation cephalosporins. However, it is important to 

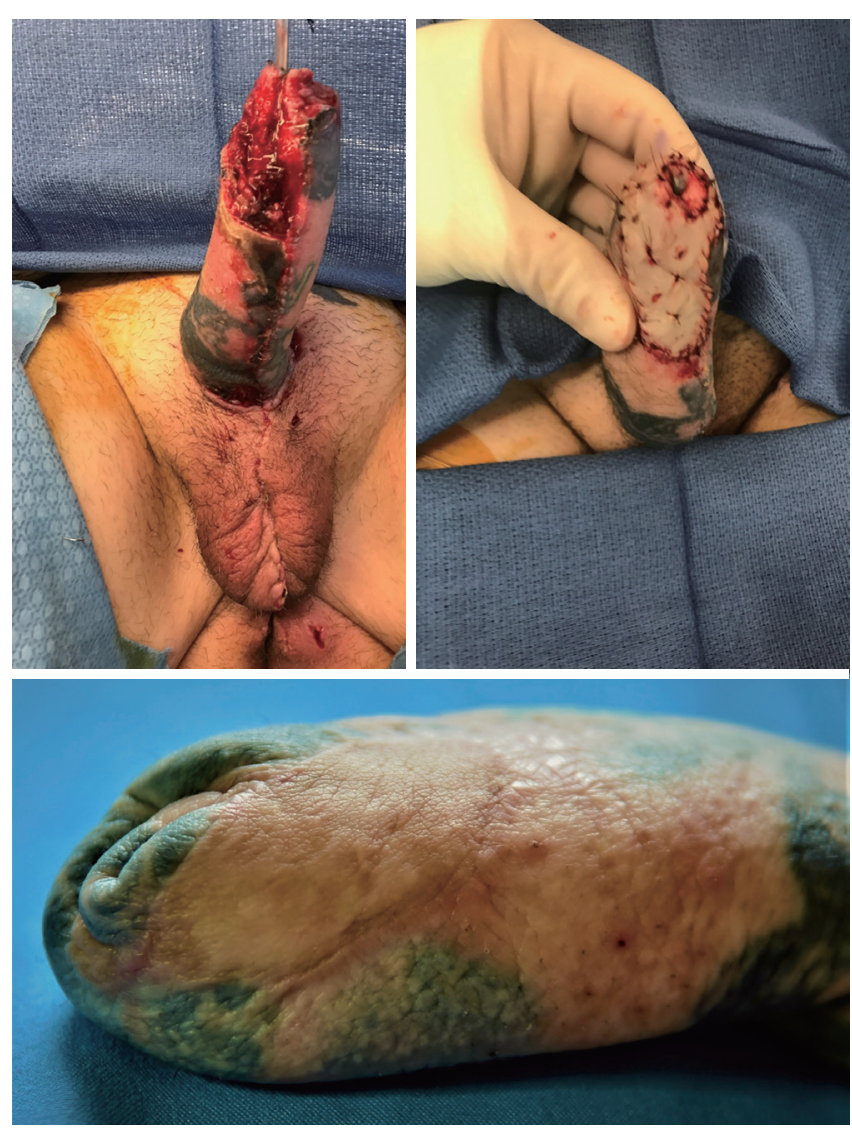

Figure 6 Partial flap loss in a radial forearm free flap. Debridement and placement of Integra followed by full thickness skingraft. Below picture is at 1 year follow up. Patient has since had successful glansplasty.

note that infections originating from the neo-urethral tissues can present in a more delayed and advanced manner. Patients may present with a normal appearing phallus that is producing malodorous or abnormal urethral discharge. We are more aggressive with these patients and will admit for broad spectrum antibiotics to prevent spread of the infection, as well as the potential urethral complications using fistulas, tissue loss, or subsequent strictures.

\section{Hematoma}

Meticulous hemostasis is essential for successful phalloplasty, as there are multiple areas that are at risk for hematoma formation. In particular, the labial tissues have a tendency to form dead space during dissection and are prone to bleeding. To reduce the risk of compressive hematomas near the pedicle, we liberally place closed suction drains in the suprapubic and groin areas. To reduce hematomas within the labial tissues, we sew in compressive bolsters over the bilateral labia majora that are left in place for 3 days.

Other important areas to consider are the mons tissues, which have a tendency to diffusely bleed even with careful dissection. Additionally, the proximal aspect of the clitoral nerve can be a source of bleeding after it is divided in preparation for nerve coaptation. We take care to cauterize the small vessels associated with the clitoral nerve to reduce this risk. Following the neurorrhaphy of the clitoral and antebrachial nerve, we routinely use fibrin glue to seal the ends of the neurorrhaphy and as an additional measure of hemostasis, apply the fibrin to the clitoral nerve stump.

At our center, we utilize the deep inferior epigastric vessels as our pedicle. Therefore, there is a potential risk of rectus sheath hematoma, however, we have not seen this. Other surgeons use the femoral vessels.

Post-operatively, surgeons must then decide on the use of medicines affecting the patient's clotting ability. For instance, aspirin for free flaps, toradol for pain control, heparin or low molecular weight heparin for DVT prophylaxis. The risk of bleeding must be carefully weighed against their benefit. We start enoxaparin or low molecular weight heparin on postoperative day 1 and get a lower extremity duplex at postoperative day 3 before the patient is allowed to ambulate.

\section{Would debiscence}

The same forces that make a phallus vulnerable to ischemia - a freely mobile flap in a dependent positionalso make it susceptible to wound breakdown. When the patient ambulates, the area is irritated by the friction, which is not well tolerated in an already moist and colonized environment. To counteract dehiscence specifically, we use long lasting deep dermal sutures and interrupted nylon sutures at the base of the flap. In their study, Monstrey et al. reported wound healing issues in $11 \%$ of phalloplasty reconstructions, but nearly all healed with conservative measures (12). This is consistent with our own experience, so long as the patient is able to clean the area regularly and monitor the wound for any signs worsening. Notably, we have seen more wound healing issues following the second stage reconstruction-either around the lateral aspects of the scrotoplasty or at the scroto-penile junction. We have moved towards using permanent sutures for all skin closures during the first and second stage, which are then removed after 3 weeks. 

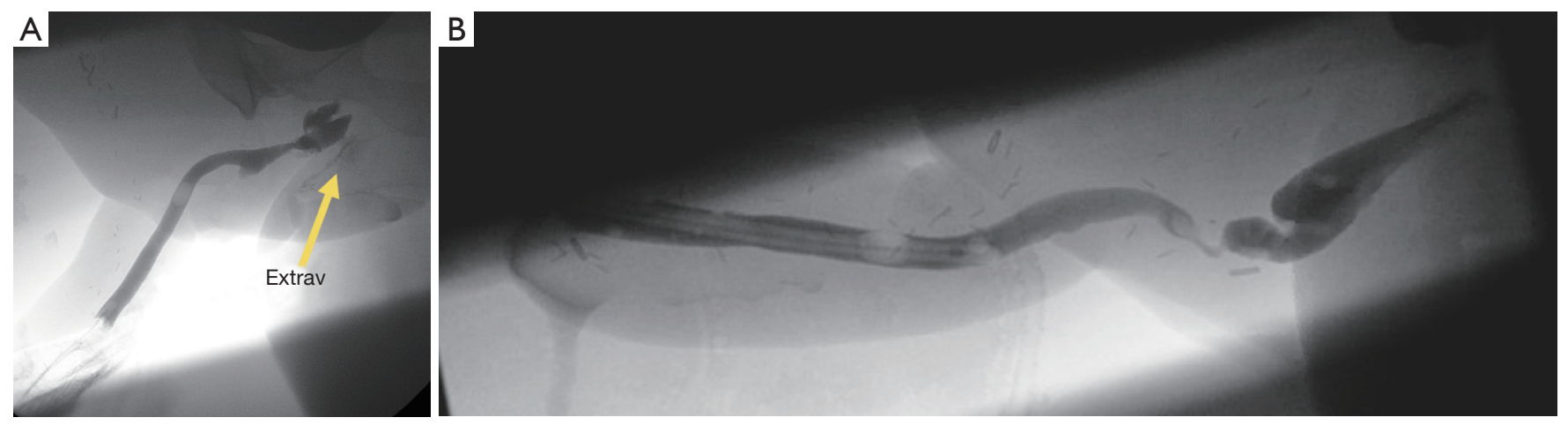

Figure 7 Retrograde urethrogram examples. (A) Retrograde urethrogram at 4 weeks after urethroplasty showing a patient with fistula; (B) retrograde urethrogram at 4 weeks after urethroplasty showing a patient with stricture.

\section{Management of urinary complications}

\section{Urinary fistula}

Fistulas can occur at various points along the reconstruction. Typical locations are the native-perineal urethral anastomosis, the perineal-penile urethral anastomosis, and along the penile shaft urethra. Management depends on the etiology of the fistula. If a distal stricture exists then urinary diversion through a suprapubic catheter and delayed stricture is performed. If it is an isolated fistula the patient may urinate from tip and fistula and return for a delayed single stage fistula repair a few months later once the scarring has matured and the fistula tract is epithelialized (see Figure 7A).

\section{Stricture}

We will defer to the separate chapter on stricture management for best practices. In brief: strictures occur at watershed areas of decreased blood supply or due to the presence of circumferential urethroplasty suture line. At this point there is no definitive evidence to suggest which reconstruction is superior, but it is conceivable that staged reconstructions allow for less ischemia and may be able to avoid circumferential scar. Similarly, stricture repair in a staged fashion with buccal graft $v s$. direct repair is debated, though in our center we use a staged approach in most cases (see Figure 7B).

\section{Post-void dribble}

A common complaint after phalloplasty is a post-void dribble. A phallus reconstruction consists of skin and fat and does not contain the elastic capability of the native urethra or the tissue analogous to the bulbospongiosus muscle. The patients may need to manually express the urine to the tip. Despite the best efforts some urine may remain. Especially in larger reconstructions such as the ALT TWT there is a considerable amount of urethral length and the larger flap is less amenable to manual compression. This should be discussed with the patient at their preoperative consultation.

\section{Retained vaginal mucosa}

The vaginectomy can be performed through a formal colpectomy and colpocleisis or via simple fulguration of the vaginal canal. The latter is assumed to lead to higher rates of retained mucosal cell islands and potential for mucocele formation leading to need for revision surgeries.

\section{Diverticula formation at proximal urethral anastomosis (internal fistula)}

The transition between native urethra and urethral lengthening is a particularly difficult area to surgically create. Often an anterior vaginal flap is utilized for this purpose. Despite best efforts this area is a weak point and while there may be no fistula formation to the outside there can be a formation of a diverticulum towards the vaginectomy site. This may be asymptomatic or lead to increase in post-void dribble, infections or pelvic pain necessitating revision surgery.

\section{Conclusions}

The wide spectrum of patient desired outcomes in 
phalloplasty means that there is no singular "gold standard" operation. Rather, surgeons are currently required to mix and match a large number of surgical techniques in order to produce an ideal aesthetic and functional outcome. This cloudiness adds unnecessary difficulty to an already major surgical procedure. The increasing frequency of GCS surgery should allow for future research that focuses on comparing long term outcomes in large cohorts of patients. With this data, it may be possible to determine more concrete guidelines that allow for patients and surgeons to have more certainty in determining their operative plans.

\section{Acknowledgments}

None.

\section{Footnote}

Conflicts of Interest: The authors have no conflicts of interest to declare.

\section{References}

1. Garaffa G, Christopher NA, Ralph DJ. Total phallic reconstruction in female-to-male transsexuals. Eur Urol 2010;57:715-22.

2. Perovic SV, Djordjevic ML. Metoidioplasty: a variant of phalloplasty in female transsexuals. BJU Int 2003;92:981-5.

3. Massie JP, Morrison SD, Wilson SC, et al. Phalloplasty with Urethral Lengthening: Addition of a Vascularized Bulbospongiosus Flap from Vaginectomy Reduces Postoperative Urethral Complications. Plast Reconstr

Cite this article as: Heston AL, Esmonde NO, Dugi DD 3rd, Berli JU. Phalloplasty: techniques and outcomes. Transl Androl Urol 2019;8(3):254-265. doi: 10.21037/tau.2019.05.05
Surg 2017;140:551e-8e.

4. Salgado CJ, Nugent AG, Moody AM, et al. Immediate pedicled gracilis flap in radial forearm flap phalloplasty for transgender male patients to reduce urinary fistula. J Plast Reconstr Aesthet Surg 2016;69:1551-7.

5. Garaffa G, Ralph DJ, Christopher N. Total urethral construction with the radial artery-based forearm free flap in the transsexual. BJU Int 2010;106:1206-10.

6. Salgado CJ, Fein LA, Chim J, et al. Prelamination of Neourethra with Uterine Mucosa in Radial Forearm Osteocutaneous Free Flap Phalloplasty in the Femaleto-Male Transgender Patient. Case Rep Urol 2016;2016:8742531.

7. Morrison SD, Shakir A, Vyas KS, et al. Phalloplasty: A Review of Techniques and Outcomes. Plast Reconstr Surg 2016;138:594-615.

8. Felici N, Felici A. A new phalloplasty technique: the free anterolateral thigh flap phalloplasty. J Plast Reconstr Aesthet Surg 2006;59:153-7.

9. Wei FC, Jain V, Celik N, et al. Have we found an ideal soft-tissue flap? An experience with 672 anterolateral thigh flaps. Plast Reconstr Surg 2002;109:2219-26; discussion 2227-30.

10. Jun MS, Pusica S, Kojovic V, et al. Total Phalloplasty With Latissimus Dorsi Musculocutaneous Flap in Female-tomale Transgender Surgery. Urology 2018;120:269-70.

11. Esmonde N, Bluebond-Langner R, Berli JU. Phalloplasty Flap-Related Complication. Clin Plast Surg 2018;45:415-24.

12. Monstrey S, Hoebeke P, Selvaggi G, et al. Penile reconstruction: is the radial forearm flap really the standard technique? Plast Reconstr Surg 2009;124:510-8. 